\begin{tabular}{|c|c|}
\hline & $\begin{array}{l}\text { International Journal of Trend in Scientific } \\
\text { Research and Development (IJTSRD) }\end{array}$ \\
\hline $\begin{array}{lll}2 \\
3\end{array}$ & International Open Access Journal \\
\hline 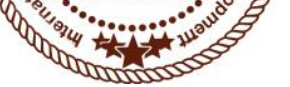 & ISSN No: 2456 - 6470 | www.ijtsrd.com | Volume - 2 | Issue -4 \\
\hline
\end{tabular}

\title{
A Study on Content-Based Image Retrieval
}

\author{
${ }^{1}$ Shabnam Kumari, ${ }^{2}$ Reema, ${ }^{3}$ Yashika Kadian \\ ${ }^{1,2}$ A.P, ${ }^{3}$ M.Tech Scholar \\ ${ }^{1,2,3}$ Department of CSE, Sat Kabir Institute of Technology \& Management, \\ Bahadurgarh, Haryana, India
}

\begin{abstract}
The development of Internet causes an eruptive expansion of digital images, and also gives people more ways to get those images. Because the dissemination of video and image data in digital form has grown, Content Based Image Retrieval (CBIR) has become an eminent research topic. The importance of an effective technique in searching and retrieving images from the huge collection cannot be overemphasized. Therefore an important problem that needs to be addressed is fast retrieval of images from large databases. To perceive images that are perceptually similar to a query image, image retrieval systems attempt to search through a database. Content-based image retrieval utilizes representations of features that are automatically extracted from the images themselves.
\end{abstract}

Keywords- CBIR, CCM, GLCM, feature vector.

\section{INTRODUCTION}

Images were traditionally superintended by first elucidating their contents and then exerting textretrieval techniques to index them. However, with the swell of information in digital image format some downsides of this technique were ejaculated:

1. Manual elucidation requires enormous amount of labor

2. Different people may perceive differently the contents of an image; thus no objective keywords for search are defined

A new research field was born in the 90's: Contentbased Image Retrieval aims at indexing and retrieving images based on their visual contents.

Also known as Query By Image Content (QBIC), presents the technologies allowing to organize digital pictures by their visual features. They are based on the application of computer vision techniques to the image retrieval problem in large databases. Content-Based Image Retrieval (CBIR) consists of retrieving the most visually similar images to a given query image from a database of images.

The shortcomings of these systems are due both to the image representations they use and to their methods of accessing those representations to find images. The problems of image retrieval are becoming widely recognized, and the search for solutions an increasingly active area for research and development.

In content-based visual retrieval, there are two fundamental challenges:

Intention gap: The intention gap refers to the difficulty that a user suffers to precisely express the expected visual content by a query at hand, such as an example image or a sketch map. The expectation of users for huge amount of objects to search among. [14]

Semantic gap: The issue related to the Semantic gap where it means the lack of coincidence between the information that one can extract from the visual data and the interpretation that the same data have for a user in a given situation. The user wants to seek semantic similarity, but the database can only provide similarity by data processing. [15] The semantic gap originates from the difficulty in describing high-level semantic concept with low-level visual feature [1] [2] [3].

\section{A. Open issues:}

1. Gap between low level features and high-level semantics

2. Human in the loop - interactive systems

3. Retrieval speed - most research prototypes can handle only a few thousand images.

4. A reliable test-bed and measurement criterion. 


\section{LITERATURE REVIEW}

Most of the search engines (ex.google, yahoo, etc.,) are based on a semantic search, i.e., the user types in a series of keywords and the images are also annotated using keywords. Thus the match is done primarily through these keywords. In the recent years CBIR system have been developed to handle the large image database effectively. Basically color, texture and shape have been used for extracting similar images from an image database. Different CBIR techniques have adopted different techniques.

Kamlesh Kumar (2016) [4] et.Al proposes CBIR method utilising Gray Scale Weighted usual system for reducing the characteristic vector dimension. The proposed procedure is more suitable for color and texture image feature analysis as in comparison with color weighted natural system as illustrated in literature review. To show the effectiveness of retrieval approach, two common benchmark dataset particularly, Wang and Amsterdam Library of Texture Images (A LOT) for color and texture had been chosen to evaluate the procedure retrieval accuracies as good as efficiencies generated by each method.

Ekta Gupta (2015) [5] et.al presents the CBIR, using facets like colour and texture, called WBCHIR (Wavelet Based color Histogram Image Retrieval).The shape and shade elements are extracted within the direction of wavelet transformation and colour histogram and the association of these elements is lively to scaling and conversion of objects in image.

Kavita Chauhan (2015) [6] et.al development of digital images requires enhanced and proficient techniques for sorting, browsing and seeking operations through ever-growing image databases. CBIR systems are search engines for image databases, which perform indexing on images according to their content and features. This paper presents the systematic review of various existing CBIR systems and their feature extraction techniques. Further the performance analysis and limitations of these systems have been discussed.

Kannan in 2010 [7] In this paper author describes that image mining is the main concept which can extract potential information from the collection. For color based image extraction RGB model is used, RGB component taken from each and every image. Images are stored by mean values of Red, Green, blue components of target images. The top ranked images are further regrouped according to texture features.
The gray level co-occurrence matrix (GLCM) used texture calculations (contrast, dissimilarity, homogeneity). The images are classified into clusters with the help of GLCM based on Low texture, average texture and high texture. Texture based classification is simply easy and efficient for real time applications as compared to Entropy method. The authors also evaluate the performance with the help of precision $\mathrm{v} / \mathrm{s}$ recall graph. Recall value 1 just by retrieving all images and precision value kept in a higher value by retrieving only few images.

Silakari in 2009 [8] In this paper a framework of unsupervised clustering of images based on the color feature of image. Clustering of images based on color moment and Block Truncation Coding to extract features from an image database is proposed. Kmeans clustering algorithm is conducted to group the dataset in various clusters.

Amanbir Sandhu, Aarti Kochhar in 2012 [9] Presents a technique for content based image retrieval using texture, color and shape for image analysis. In this paper they worked with the three features i.e. texture, color and shape and its different combinations. The GLCM is used for texture feature extraction, histogram for Color feature extraction and for shape different factors are found like area, Euler No., eccentricity and Filled Area.

Choras et al. [10] proposed an integrated color, texture and shape feature extraction method in which Gabor filtration is used for determining the number of regions of interest (ROIs). They calculated texture and color features from the ROIs based on threshold Gabor features and histograms, color moments in YUV space, and shape features based on Zernike moments. The features presented proved to be efficient in determining similarity between images.

Maheshwari et al. [11] have used Color moment and Gabor filter to extract features for image dataset. In their study, K-means and hierarchical clustering algorithm is applied to group the image dataset into various clusters.

In [12] a novel CBIR system based on three methods: Initially co-occurrence matrix (CCM) is computed for color feature. The CCM matrix is used to analyze probability of occurrence of pixels having same color and the adjacent pixels in the image. Second the difference between pixels of scan patterns (BDPSP) is computed to find out the variance among all pixels of scan patterns. The third method is color distribution for K-mean algorithm. It is based on color histogram 
in which each color pixel is substituted by any color that is utmost related to the existing color. The Kmean algorithm divides all the pixels into k clusters. A CBIR system based on three algorithms viz. feature extraction, image mining and the rule based is proposed in [13]. The first algorithm globally extracted the color and texture features from the image. It is considered that these features are invariable to the image transform and could be used for the detection of the objects. The second algorithm uses the image mining method that implies clustering algorithm to retrieve hidden knowledge from the image. The third algorithm uses the rules based on relevance feedback to filter the results and to improve the clusters.

\section{OVERVIEW OF CBIR SYSTEM}

Content-based image retrieval uses the visual contents of an image such as texture, color, shape, and spatial layout to represent and index the image. In typical CBIR systems, the visual content of the images in the database are extracted and described by multidimensional feature vectors. The feature vector of the images in the database forms a feature database. To retrieve the images, users provide the retrieval system with example images. The system then changes these examples into its internal representation of feature vectors.

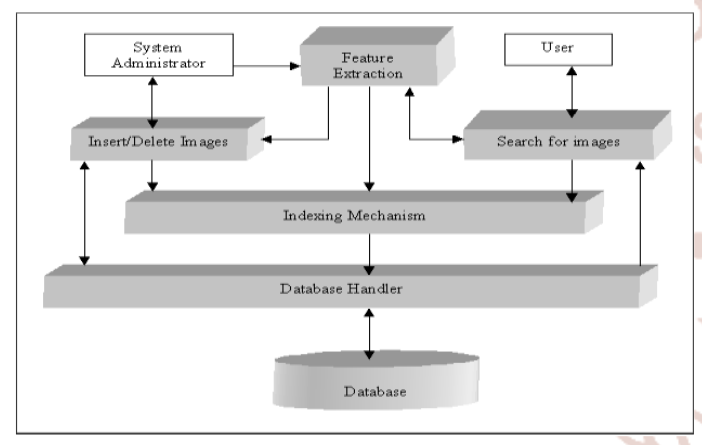

Figure 1: Overview of CBIR System

The diagram above describes the overview of the Content Based Image Retrieval (CBIR) system. Each block of the figure describes a particular process in the system. As shown in the figure all the feature vectors of the images are stored in the databases called as the feature database, the corresponding feature vectors of query image is extracted and it is compared with all the feature vectors stored in the database using a suitable similarity measurement technique and relevant images are retrieved on the basis of predefined threshold.

\section{A. Components of CBIR System}

The CBIR system consists of the following components:

\section{a. Query image}

It is the image to be found in the image database, whether the similar image is present or not. And how many are similar kind images are exist or not.

\section{b. Image database}

It consists of n number of images depends on the user choice.

\section{c. Feature extraction}

It separates visual information from the image and saves them as features vectors in a features database. The feature extraction finds the image detail in the form of feature value (or a set of value called a feature vector) for each pixel. These feature vectors are used to compare the query image with the other images and retrieval.

\section{d. Image matching}

The information about each image is stored in its feature vector for computation process and these feature vectors are compared with the feature vectors of query image which helps in measuring the similarity.

\section{e. Resultant retrieved images}

It finds the previously maintained information to find the matched images from database. The output will be the similar images having same or closest features as that of the query image.

\section{FEATURE VECTORS}

In pattern recognition and machine learning, a feature vector is an n-dimensional vector of numerical features that represent some object. Many algorithms in machine learning require a numerical representation of objects, since such representations facilitate processing and statistical analysis. When representing images, the feature values might correspond to the pixels of an image, when representing texts perhaps to term occurrence frequencies.

Some important feature vectors of the image are as follows:

1. Mean: Mean gives the overall distribution of the pixel's gray level.

2. Variance: The variance is a measure of dispersion. It tells us something about the scatter of scores (here pixels) around the mean. It is 
defined as the mean squared deviation from the mean, and symbolized by a small sigma squared. Its formula is:

Variance $=\sigma \times 2=\sum(\mathrm{X}-\mathrm{M}) 2 / \mathrm{N}$

Where $\mathrm{X}=$ Pixel value

$\mathrm{M}=$ Mean of the Image

$\mathrm{N}=$ Total number of pixel

\section{Standard Deviation:}

The standard deviation is the square root of the variance and is Symbolized by a small Greek sigma - $\sigma$. Its formula is the square root of any of the formulae for the variance, e.g. $x=\sum(x) 2 / N$

\section{Entropy:}

Entropy is a statistical measure of randomness that can be used to characterize the texture of the input image.

5. Texture:

Texture is that innate property of all surfaces that describes visual patterns, each having properties of homogeneity. It contains important information about the structural arrangement of the surface, such as; clouds, leaves, bricks, fabric, etc. It also describes the relationship of the surface to the surrounding environment. In short, it is a feature that describes the distinctive physical composition of a surface.

Texture properties include: Coarseness, Contrast, Directionality, Line-likeness, Regularity, and Roughness in the image.

Texture is one of the most important defining features of an image. It is characterized by the spatial distribution of gray levels in a neighborhood. In order to capture the spatial dependence of gray-level values, which contribute to the perception of texture, a two-dimensional dependence texture analysis matrix is taken into consideration. This two-dimensional matrix is obtained by decoding the image file; jpeg, bmp, etc.

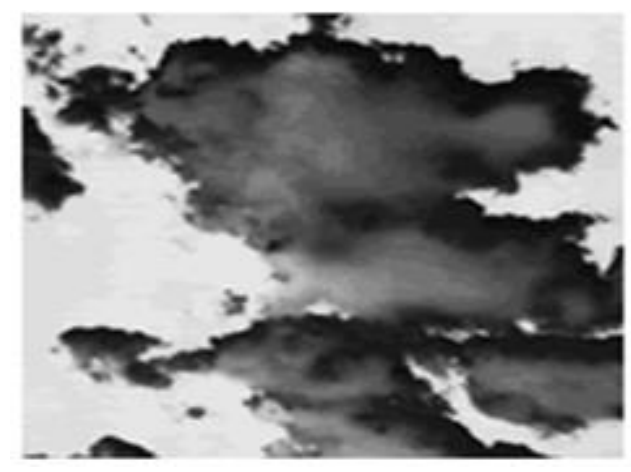

(a) Clouds

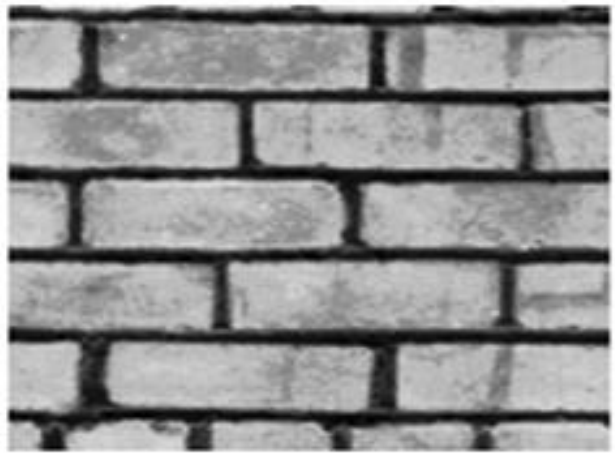

(b) Bricks

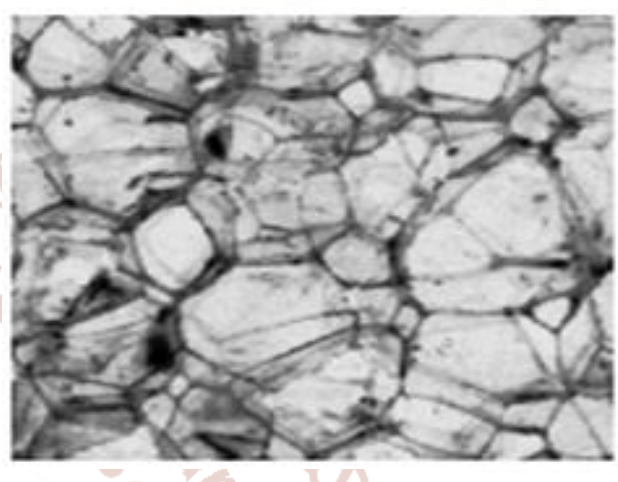

(c) Rocks

Figure 2: Examples of Textures

6. Color :

One of the most important features that make possible the recognition of images by humans is color. Color is a property that depends on the reflection of light to the eye and the processing of that information in the brain. We use color every day to tell the difference between objects, places, and the time of day. Usually colors are defined in three dimensional color spaces. These could either be RGB (Red, Green, and Blue), HSV (Hue, Saturation, and Value) or HSB (Hue, Saturation, and Brightness). The last two are dependent on the human perception of hue, saturation, and brightness.

7. Shape :

Shape may be defined as the characteristic surface configuration of an object; an outline or contour. It permits an object to be distinguished from its surroundings by its outline. Shape representations can be generally divided into two categories:

- Boundary-based, and

- Region-based. 


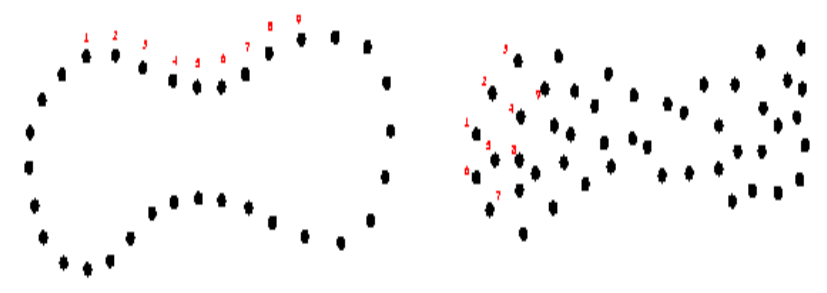

Figure 3: Boundary-based \& Region-based

\section{WORKING}

1. CBIR provides the retrieval of the digital images similar to the query image from the large storage of the database according to their content.

2. When user gives the query image Gabor transform is applied over the image.

3. Features (Mean, Entropy \& Standard Deviation) of the transformed are calculated.

4. Next the query image is split into R, G and B plane.

5. Features (Mean, Entropy \& Standard Deviation) of each plane are calculated again.

6. Query image is then transformed with discrete wavelet Transform (DWT) 1-level.

7. Again, the features of the each component of transformed image are calculated.

8. Now the same procedure is applied on each image of the database.

9. The Feature Vector of the database images (Fd) is obtained.

10. The Feature Vector of the query image $(\mathrm{Fq})$ is obtained.

Similarity measurement using Euclidean distance is done between the feature vectors of database \& query image.

Images whose distance is more than the predefined threshold is retrieved from the image database.

\section{CONCLUSION}

CBIR is a fast developing technology with considerable potential. The dramatic progress by digital media at home, in enterprises, and on the web, has from above her last decade spawned great interests in developing ways for powerful indexing and searching of desired visual contents to open the worth of those contents. CBIR is the sub concern of CBR it is necessary to advance energy instruments to retrieving images from the web where the number and size of digital snap sort is developing fast. The area of content-based image retrieval is a hybrid research area that requires knowledge of both computer vision and of database systems. There are various applications of CBIR in every fields of life like blood cell detection, archeology, criminal investigation, image search, social networking sites, forensic Labs, and satellite etc. The field appears to be generating interesting and valid results, even though it has so far led to few commercial applications.

\section{References}

1) Alzubi, A. Amira, and N. Ramzan, "Semantic content-based image retrieval: A comprehensive study," Journal of Visual Communication and Image Representation, vol. 32, pp. 20-54, 2015

2) X. Li, T. Uricchio, L. Ballan, M. Bertini, C. G. Snoek, and A. D. Bimbo, "Socializing the semantic gap: A comparative survey on image tag assignment, refinement, and retrieval," ACM Computing Surveys (CSUR), vol. 49, no. 1, p. 14, 2016.

3) Z. Lin, G. Ding, M. Hu, and J. Wang, "Semanticspreserving hashing for cross-view retrieval," in IEEE Conference on Computer Vision and Pattern Recognition (CVPR), 2015, pp. 3864-3872.

4) Mohammed Alkhawlani and Mohammed Elmogy, "Textbased, Content-based, and Semantic-based Image Retrievals: A Survey" Volume 04 - Issue 01, January 2015

5) Kavita Chauhan and Shanu Sharma, "A Review on Feature Extraction Techniques for CBIR System" Accepted 31- July - 2015 Article ID ICIEMS018 eAID ICIEMS.2015.018 Received 10 - July - 2015

6) L. Haldurai, V. Vinodhini, "A Study on Content Based Image Retrieval Systems" International Journal of Innovative Research in Computer and Communication Engineering -2015.

7) A.Kannan,Dr.V.Mohan,Dr.N.Anbazhagan, "Image Clustering and Retrieval using Image Mining Techniques", IEEE International Conference on Computational Intelligence and Computing Research, 2010.

8) Dr. Sanjay Silakari, Dr. Mahesh Motwani and Manish Maheshwari, "Color Image Clustering using Block Truncation Algorithm", IJCSI International Journal of Computer Science Issues, Vol. 4, No. 2, 2009.

9) Amanbir Sandhu, Aarti Kochhar, "Content Based Image Retrieval using Texture, Color and Shape for Image Analysis", International Journal of Computers \& Technology, Volume 3, No. 1, AUG, 2012.

10) Choras, R. 2003. "Content-based image retrieval using color, texture, and shape information", In. Sanfeliu, Riuz-Shulcloper J. (eds) Progress in 
pattern recognition, speech and image analysis.

Springer, Heidelberg

11) Maheshwari, M. , Silakari, S. and Motwani, M. 2009. "Image Clustering using Color and Texture", Computational Intelligence, Communication Systems and Networks, pp. 403408.

12) Y. C. C.H. Lin, R.T. Chen, "A smart contentbased image retrieval system based on color and texture feature," Image Vis. Comput., vol.27 (6), p. 658-665., 2009

13) A. del Bimbo, "Visual information retrieval," Morgan Kaufmann Publishers, 1999.

14) Gaurav Jaswal Amit Kaul , " Content Based Image Retrieval ", National Conference on Computing, Communication and Control, A Literature Review, National Institute of Technology, Hamirpur-177001, Himachal Pradesh(India).

15) R.Senthil Kumar, Dr. M. Senthilmurugan, "Content-Based Image Retrieval System in Medical",International Journal of Engineering Research \& Technology (IJERT),Vol. 2 Issue 3, March - 2013,ISSN: 2278-0181.

16) Howarth, P. and Ruger, S. "Evaluation of texture features for content based image retrieval", In: Enser P. et al. (eds) Image and video retrieval. Springer LNCS 3115:pp.326-334.

17) Choras, R.S., Andrysiak, T. and Choras, M. 2007. "Integrated color, texture and shape information for content-based image retrieval", Pattern Anal Applic. 10: 333-343.

18) Huang, Z.C., Chan, P.P.K., Ng, W.W.Y., Yeung, D.S. 2010. "Content-based image retrieval using color moment and Gabor texture feature", in Poceedings of the IEEE Ninth International Conference on Machine Learning and Cybernetics, Qingdao, pp. 719-724.

19) Danish, M., Rawat, R., \& Sharma, R. (2013). A Survey: Content Based Image Retrieval Based On Color, Texture, Shape \& Neuro Fuzzy. Int. Journal Of Engineering Research And Application, 3(5), 839-44

20) Jaswal, G., \& Kaul, A. (2009). Content Based Image Retrieval - A Literature Review. In Communication and Control. India: National Institute of Technology. 